\title{
Examining Patient and Provider Experiences with Integrated Care at a Community Health Clinic
}

\author{
Rachel A. Petts, PhD \\ Rhonda K. Lewis, PhD, MPH \\ Keyondra Brooks, PhD \\ Sarah McGill, MA \\ Teresa Lovelady, MSW, MBA \\ Mel Galvez, MBA \\ Erica Davis, MS
}

\begin{abstract}
The purpose of this study was to examine patient and provider experiences of integrated behavioral health care at a Federally Qualified Health Center (FQHC). Using a mixed methodology design, both patients $(n=186)$ and providers $(n=17)$ completed a survey regarding satisfaction with care and the extent of integration at the clinic, as well as attended a focus group or interview $(n=11$ patients; $n=12$ providers) regarding their satisfaction and experiences. Both patients and providers found integration to be acceptable and satisfactory and the integration of services among different health care providers occurred fairly regularly. Themes from the provider and patient interviews/ focus groups highlighted both positive aspects of the integration and specific challenges within the clinic. This more nuanced perspective of integration both replicates and extends upon previous research regarding satisfaction with integrated care and emphasizes the complexities and challenges of integration within community health clinics.
\end{abstract}

Address correspondence to Rhonda K. Lewis, PhD, MPH, Department of Psychology, Wichita State University,

Wichita, KS, USA.rhonda.lewis@wichita.edu.

Keyondra Brooks, PhD, Department of Psychology, Wichita State University, Wichita, KS, USA.

Sarah McGill, MA, Department of Psychology, Wichita State University, Wichita, KS 67260, USA.

Rachel A. Petts, PhD, School of Psychology and Counseling, Fairleigh Dickinson University, 1000

River Road, Teaneck, NJ 07666, USA.

Teresa Lovelady, MSW, MBA, HealthCore Clinic, Wichita, KS, USA.

Mel Galvez, MBA, HealthCore Clinic, Wichita, KS, USA.

Erica Davis, MS, HealthCore Clinic, Wichita, KS, USA.

The Journal of Behavioral Health Services \& Research, 2022. 32-49. () 2021, National Council for Behavioral Health, DOI 10.1007/s11414-021-09764-2 


\section{Keywords Integrated care - Behavioral health - Federally Qualified Health Centers · Patient satisfaction}

Since its inception in 1965, the federally qualified health center (FQHC) has served as the primary safety net health system in the USA. ${ }^{1}$ As a federally supported institution, the FQHC's main goal is to provide comprehensive and accessible high quality health care for underserved communities. As of 2018, there are over 1300 FQHCs serving more than 28 million patients. ${ }^{2}$ The majority of these patients live well under the poverty line and many are racial/ethnic minorities and/or special populations (e.g., unsheltered, migrant/seasonal workers) who typically lack access to affordable and culturally competent health care. Notably, FQHCs provide care for a growing number of patients with chronic health diseases. According to 2018 reporting, this has included over 6 million patients overweight or with obesity, 4.7 million with hypertension, and 2.5 million with diabetes. ${ }^{2}$ During the COVID-19 pandemic, FQHCs have expanded their roles to promote access to testing and vaccine distribution to the broader community, while maintaining essential health services for their clinic population. In short, FQHCs serve the most medically, economically, and often socially vulnerable populations and consequently are at the forefront of implementing innovative health service delivery models aimed at improving access to, and quality of, health care.

One such innovative health service model is integrated care. Integrated care refers to the integration of multiple health care services (often behavioral health and medical), under one roof and with a unified care plan. ${ }^{3}$ While the typical clinic's main goal is to provide primary health care for all ages and conditions (with preventative care and management of illness and chronic conditions the foundation of these services), a core objective of the FQHC is to be comprehensive in the services offered and to provide, or facilitate referral to, other pertinent health care providers. These often include health services that, while not considered primary care, are essential for disease prevention and health promotion, such as dentistry and behavioral health/substance use services. Additionally, many clinics provide transportation, patient education/outreach, and other care management services to increase accessibility and engagement with health care. Indeed, while medical services made up the vast majority of clinic visits in 2018 (66.58\%), dental services made up 14.27\%, mental health $9.33 \%$ and care management/patient education $5.62 \%$, indicating that integration may be occurring in a large number of clinics. ${ }^{2}$ The impetus to provide services other than medical care in FQHCs is not new, but the passing of the Affordable Care Act (ACA) has provided much needed financial incentive to promote integrated services, while many government agencies (e.g., HRSA) continue to fund and endorse coordinated and integrated care. ${ }^{4}$

The majority of integrated care models have focused on the merging of health and behavioral health services. While there are several approaches to this type of integration, the most prominent is the primary care behavioral health model $(\mathrm{PCBH})$, which involves coordination among health care providers exemplified by warm hand-offs, in which a provider (typically medical) hands off services to another member of the health care team (e.g., behavioral health) for a same-day service and real-time consultation. ${ }^{5,6}$ For instance, an individual with poor diabetes management and depression who sees their primary care provider for a medical check-up may also be referred to a behavioral health provider that day to discuss behavioral strategies to improve medication adherence and mood. One can imagine how this service delivery model could be applied to other members of the team-for instance, same day consults with a dental hygienist for a patient who also needs a dental filling or a brief check in with the patient education specialist to discuss diet and exercise. Generally, the type of service provided is brief (e.g., $30 \mathrm{~min}$ ) and time limited in nature (perhaps $1-3$ visits). ${ }^{5,6}$ Behavioral health providers in these settings often act like primary care providers in that they are generalists and will see any mental health issue and/or health condition influenced by psychological or social factors. 
There are many advantages to PCBH models, including increased access and engagement in mental health care, increased patient satisfaction, and improved patient health outcomes. ${ }^{7,8}$ Although the majority of research has focused on academic medical centers or Veterans Affairs medical centers, there is growing evidence of PCBH implementation in FQHCs and associated outcomes. For instance, PCBH has been associated with increased utilization of medical services and decreased hospitalizations for individuals with severe mental illness and has also been shown to promote access to mental health services particularly in the underserved populations that FQHCs typically provide for. ${ }^{9,10}$ Further, a recent randomized trial comparing integration to usual care found that the model improved quality of care and access to preventative services in a sample of patients with severe mental illness; however, it did not differentiate from usual care in patient health outcomes. ${ }^{11}$ While obtaining patient and service level outcomes is important, assessing provider perceptions is also necessary to help improve implementation and dissemination of integration in the community. Qualitative research has also examined interdisciplinary provider perspectives, highlighting both benefits and challenges of integration and providing more nuanced recommendations for how to enhance implementation of integrated care. ${ }^{12}$

Although the majority of integrated care research in FQHCs focuses on the integration of behavioral health and medical services, there is also growing evidence supporting the integration of oral care and pharmacy among other services. ${ }^{13-15}$ Interestingly, there is a lack of research on all aspects of integration that are unique to many FQHCS - that is, the integration of health, behavioral health, dental services, pharmacy, care coordination, or any other health services. The integration of several services is a strength of FQHCs and an important aspect of the model to evaluate, particularly given its potential to reduce health inequities that have been further exacerbated by the COVID-19 pandemic. Given the dearth of research within this area, examining patient and provider experiences of a more complete integrated clinic may be a worthy first step.

The overarching goal of this study was to examine patient and provider experience of a fully integrated FQHC that offered several services: medical, dental, behavioral health, pharmacy, and care management. The unique aspects of this evaluation relate to its breadth and method of study: both patient and provider satisfaction were obtained for all services offered within the clinic, and qualitative and quantitative methodology were used while working with community partners throughout the process. Community-based participatory research (CBPR) was the guiding framework used in this study. ${ }^{16}$ In this model, researchers and community partners are co-researchers and are involved in every step of the process which includes planning the study design, developing the surveys, and disseminating the results. A community partnership was formed between a local FQHC and a midsized midwestern university, and the FQHC received funding to build capacity related to implementing their vision of integrated health care. The local university researchers had a long-standing relationship with the clinic since its inception and agreed to work together to assess how well the new model was working. The primary research questions were as follows:

1. What was the current status of integration at the clinic as reported by providers (i.e., how well was integration occurring or not occurring at all levels?)

2. How satisfied were providers with the model of integration?

3. What was the current status of integration as reported by patients?

4. How satisfied were patients with the services provided by the integrated clinic? Relatedly, what factors predicted satisfaction of services by patients? 


\section{Method}

\section{Design and setting}

This study utilized a mixed methodology design. Qualitative and quantitative measures were used to evaluate patient and provider satisfaction with the model and to measure levels of integration. Data were collected in 2019 at an urban, midwestern integrated care practice offering behavioral health, dental, medical, pharmacy, and education and outreach services. The clinic has been a Federally Qualified Health Center (FQHC) for over a decade, and became a Patient Centered Medical Home (PCMH) when integrating behavioral health services on-site in 2013. The integrated care model implemented in the clinic is based upon the PCBH model with behavioral health providers (typically Master's level clinicians) embedded within the primary care team, providing same day consultations and brief interventions to any patient with behavioral health or health-related needs. These behavioral health providers also provided brief follow-up services and helped facilitate referral to traditional outpatient services. Dental services were offered same day by dental hygienists or a dentist via a consultation model, although they also operated their own scheduled appointments and follow-up. Lastly, education and outreach services are available to patients via referral and a pharmacy was located within the building, with the pharmacist available for some consultation services. While there were no incentives for providers to initiate same day consultations, or warm "hand-offs", with different members of the team, all providers were instructed to consult and collaborate if there was an identified need.

\section{Quantitative component}

\section{Participants and recruitment}

Patients 18 and older were recruited via a convenience sampling strategy to complete a paper questionnaire. One hundred eighty-nine adult patients voluntarily completed a paper survey in the waiting room of the clinic over a five-day period. Patients were informed that the survey was voluntary, confidential, and would have no impact on the healthcare they received at the clinic. Members of the research team affiliated with the university recruited participants. Those who completed the survey had a chance to enter a drawing to win a $\$ 50$ gift card. Table 1 presents the background characteristics of the patients who completed the survey.

Seventeen healthcare providers completed a paper survey during a lunchtime integrated healthcare meeting. Lunch was provided as an incentive. Providers were informed that the survey was voluntary and confidential. The research team associated with the university distributed the survey. Six medical providers, 7 behavioral health providers, and 4 dental providers completed the survey. Table 1 also provides demographics of the providers, including length of employment at the clinic. All providers were full-time employees of the clinic and were funded by revenue and/or grants.

\section{Patient measures}

The $P S Q-18$ is a brief measure of patient satisfaction with health care. ${ }^{17}$ Patients report their level of agreement (from Strongly Agree to Strongly Disagree) for a variety of statements that correspond with seven dimensions of satisfaction: general satisfaction, technical quality, interpersonal manner, communication, finances, convenience and accessibility, and time spent with a doctor. ${ }^{17}$ The PSQ-18 is a reliable and valid measure of satisfaction that can be used in a variety of settings and was chosen to supplement the researcher created integration satisfaction measure (see below). ${ }^{18}$ The Cronbach's alpha for the PSQ-18 in this sample was 0.90 . 
Table 1

Provider and patient demographics

\begin{tabular}{|c|c|c|c|}
\hline \multicolumn{2}{|l|}{ Provider } & \multicolumn{2}{|l|}{ Patient } \\
\hline & $\mathrm{N}=17$ & & $\mathrm{~N}=186^{\mathrm{a}}$ \\
\hline Age $M(S D)$ & $36.4(11.3)$ & Age $M(S D)$ & $44.2(15.5)$ \\
\hline Female $(\%)$ & $11(64.7 \%)$ & Female $(\%)$ & $135(72.6 \%)$ \\
\hline Race/ethnicity (\%) & & Race/ethnicity (\%) & \\
\hline White & $9(52.9 \%)$ & White & $56(30.1 \%)$ \\
\hline Black & $4(23.5 \%)$ & Black & $79(42.5 \%)$ \\
\hline Hispanic & $2(11.8 \%)$ & Hispanic & $28(15.1 \%)$ \\
\hline Asian & $1(5.9 \%)$ & American Indian or Alaskan Native & $3(1.6 \%)$ \\
\hline \multirow[t]{2}{*}{$\mathrm{Bi} /$ multi-racial } & $1(5.9 \%)$ & $\mathrm{Bi} /$ multi-racial & $11(5.9 \%)$ \\
\hline & & Other & $5(2.7 \%)$ \\
\hline $\begin{array}{l}\text { Professional/training back- } \\
\text { ground }(\%)\end{array}$ & & Education level (\%) & \\
\hline Medical & $6(35.3 \%)$ & Less than HS degree & $26(14 \%)$ \\
\hline Behavioral health & $7(41.2 \%)$ & HS degree or equivalent & $60(32.3 \%)$ \\
\hline \multirow[t]{2}{*}{ Dental } & $4(23.5 \%)$ & Some college & $59(31.7 \%)$ \\
\hline & & College degree + & $34(18.4 \%)$ \\
\hline Years worked at clinic $(\%)$ & & Years going to clinic (\%) & \\
\hline Less than 6 months & $4(23.5 \%)$ & Less than 6 months & $39(20.6 \%)$ \\
\hline 6 months-1 year & $6(35.3 \%)$ & 6 months-1 year & $33(17.5 \%)$ \\
\hline $1-2$ years & $4(23.5 \%)$ & $1-2$ years & $35(18.5 \%)$ \\
\hline $2-5$ years & $2(11.8 \%)$ & $2-5$ years & $41(21.7 \%)$ \\
\hline \multirow[t]{2}{*}{ More than 5 years } & $1(5.9 \%)$ & More than 5 years & $34(18 \%)$ \\
\hline & & Sees providers outside of clinic $(\%)$ & $68(37.2 \%)$ \\
\hline
\end{tabular}

${ }^{a}$ Some demographic data are missing; $H S$, high school

The researcher-created form consisted of several questions regarding patient experience of integration and satisfaction specific to integrated care. Patients reported their level of agreement $(1=$ Strongly Disagree to $5=$ Strongly Agree $)$ on a series of statements regarding their care at the clinic. The 11 questions related to satisfaction (see Table 2 for question prompts) had a Cronbach's alpha of 0.94, suggesting excellent internal consistency. Patients also rated their overall physical and mental health on a 1 (Poor) to 5 (Excellent) scale. The average selfreported rating of overall health was $M=3.1(S D=1.06)$, corresponding to a qualitative rating of "Good." The average self-reported rating of mental health was slightly lower, with an average of $2.8(S D=1.3)$, in between a qualitative rating of "Fair" and "Good."

\section{Provider measures}

The researcher created form consisted of several questions regarding the clinic's level of integration/collaboration and satisfaction with the model. The 9-item satisfaction measure asked providers to rate their level of agreement with a series of statements $(1=$ Strongly Disagree to $5=$ Strongly 


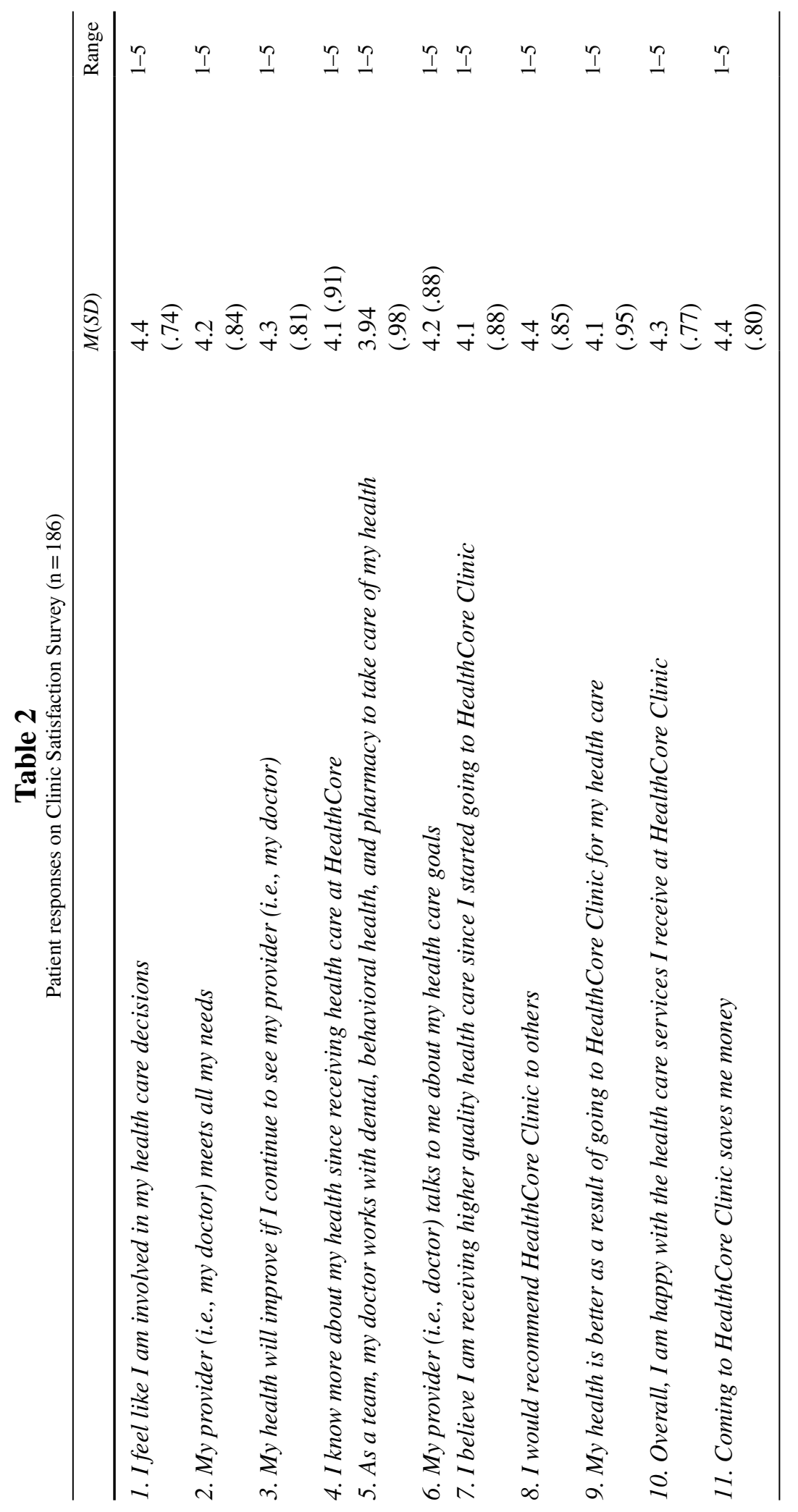


Agree) and had a Cronbach's alpha of 0.85, suggesting good internal consistency. See Table 3 for question prompts.

\section{Data analysis}

Three patient cases were dropped from the final analysis: one individual with an incomplete consent and two had significant missing data. Data were analyzed using SPSS. Descriptive statistics including frequencies, percentages, means, and standard deviations were utilized for the patientand provider-researcher created questionnaires and the PSQ-18. Further, a multiple regression was run to predict patient satisfaction on the PSQ-18 from age, gender, race/ethnicity, education level, and current ratings of health and mental health, respectively. All assumptions for the regression analysis were met.

\section{Qualitative data}

\section{Participants and recruitment}

The clinic identified patients with diabetes as a target population for the focus groups given that they are at-risk for a variety of negative health outcomes and a group who may benefit significantly from an integrated health model of care. Four semi-structured patient focus groups $(n=11)$ were conducted by the research team, with the goal of obtaining more nuanced perspectives of the integrated clinic. Questions were asked pertaining to: participant's knowledge of integrated care and patient centered models; the extent to which patients perceived that the clinic met integrated or patient centered model goals; perceived level of health and well-being since coming to the clinic; overall experiences at the clinic; and ways to improve the clinic. Given the time commitment associated with the focus groups, a snack and gift card was provided as incentive. Similar to the survey, the participants were informed that focus groups were voluntary, confidential, and would have no impact on the healthcare they received at the clinic.

A total of 12 providers (all full-time employees of the clinic) participated in semi-structured focus groups or interviews in order to obtain more specific feedback on the integrated model at the clinic. Separate focus groups were conducted for nurses, medical providers, and behavioral health providers. Due to provider schedules, three individual interviews were conducted with a pharmacist, dentist, and medical provider to ensure representation of all providers. Questions were asked pertaining to: how well the clinic was implementing the integrated care model; provider experiences with integration; comfort level of collaborating with other providers; and suggestions for how the clinic could improve. The research team completed the provider interviews/focus groups and lunch was provided as incentive. Finally, providers were informed that their participation was voluntary and confidential.

\section{Coding and analysis}

Data from the patient and provider focus groups were analyzed using an inductive semantic thematic analysis approach. ${ }^{19}$ The research team coded data using the six phases of thematic analysis outlined by Braun \& Clarke. ${ }^{19}$ The first phase included familiarization of data through listening to, transcribing, and re-reading transcripts in accordance to audio-recordings. The second phase included coding the data by individually identifying and labeling relevant features, then coming together to find agreement on codes, and codebook generation. The third phase included searching for themes by clustering codes to map key patterns in the data. The fourth phase included reviewing themes to examine if they were a good fit with the entire data set and assuring there was no 


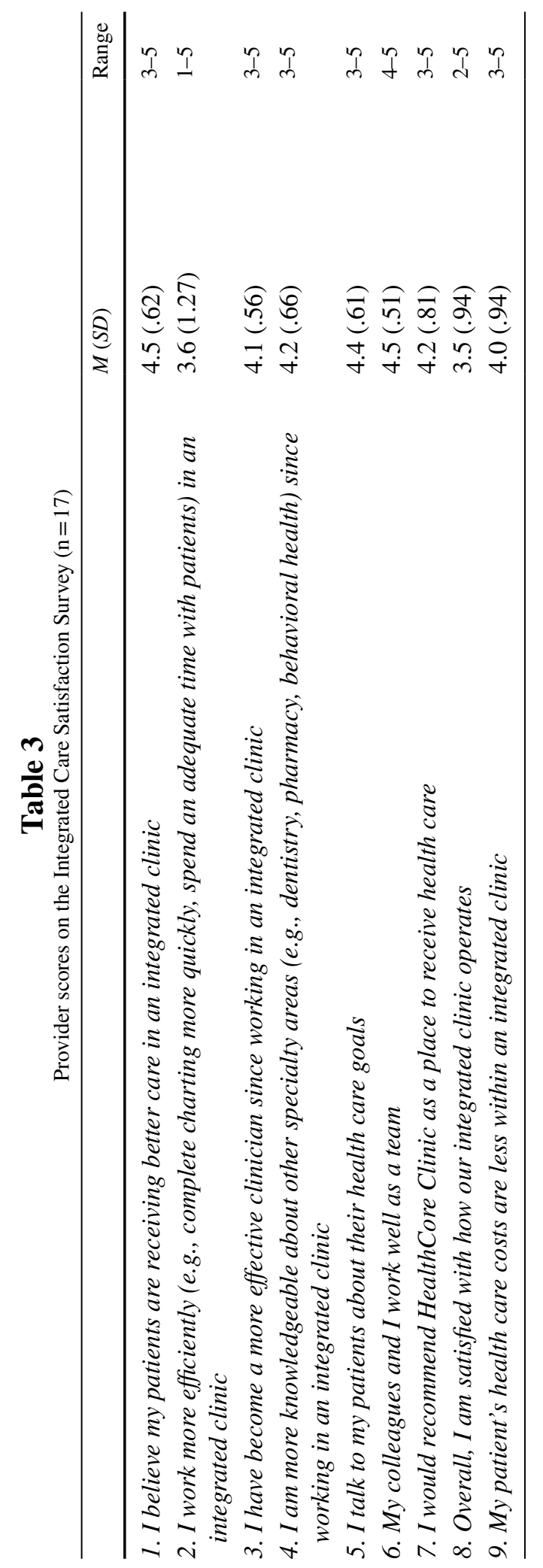


overlapping concepts among the themes. The fifth phase included the research team coming together to define and name themes; the last phase included reviewing themes for clarity and context within the larger dataset and write up. Due to the unique nature of experiences between health care providers, researchers initially completed separate coding and theming procedures for nursing, medical, behavioral health, pharmacy, and dental groups. However, given a clear overlap in themes and codes among providers, all themes were combined in the final analysis.

\section{Results}

\section{Quantitative outcomes}

\section{Provider report of integration}

Most providers reported collaborating with another provider "very often" (76.5\%), while $17.6 \%$ reported "always" and 5.9\% reported "sometimes." However, the more specific measure of integration - that is, the frequency of bringing in another provider during a visit, or a warm hand-off — was more evenly split: $35.3 \%$ of providers reported doing this "very often", $41.2 \%$ reported "sometimes", and $23.5 \%$ reported "rarely" (note that no providers reported doing this "always"). The most frequently reported collaborating providers were behavioral health and medical $(88.2 \%$ each), followed by dental (64.7\%), and pharmacy (35.3\%). Most providers reported closing the communication loop with other providers "immediately" (76.5\%) after completing a patient consultation.

\section{Provider satisfaction}

As shown in Table 3, providers exhibited a generally high degree of satisfaction with the integrated model. The highest rated items included patients receiving better care at an integrated clinic and working as a team with colleagues. There was more variability and relatively lower scores for the items pertaining to working more efficiently in an integrated clinic and overall satisfaction with how the clinic operates.

\section{Patient report of integration}

Only $36 \%$ of patients experienced an integrated visit at the clinic. However, the overall quality of these visits was rated approximately "Very Good" $(M=4.12, S D=0.98)$. Over the past year, approximately $73 \%$ of patients reported being seen by medical staff, $28.5 \%$ by dentistry, and $25.3 \%$ by behavioral health. Further, $40.9 \%$ of patients reported using the in house pharmacy.

\section{Patient satisfaction}

Table 2 shows the average scores for the integrated care specific satisfaction measure. Similar to providers, patients reported generally high levels of satisfaction with the clinic. The three relatively highest items included feeling involved in health care decisions, recommending the clinic to others, and saving money by going to the clinic. The relatively lowest rated item suggested that patients neither agreed nor disagreed that their doctor works with other members of the team to take care of their health.

On the PSQ-18, the average satisfaction score was $65.7(S D=12.1$, range 21-85), indicating a generally high level of satisfaction. The highest evaluation was in the communication subscale $(M=4.11, S D=0.84)$, followed by the interpersonal manner subscale $(M=4.10, S D=0.93)$, 
general satisfaction $(M=3.92, S D=0.97)$, technical quality $(M=3.89, S D=0.82)$, time with doctor $(M=3.82 S D=0.91)$, financial aspects $(M=3.81, S D=0.97)$, and finally, accessibility/convenience $(M=3.60, S D=0.96)$.

\section{Patient predictors of satisfaction}

The multiple regression model was significant $\mathrm{F}(10,152)=2.02, p<05$, although only accounting for only a small proportion of the variance, $\mathrm{R}^{2}=11.7 \%$ and adj $\mathrm{R}^{2}=5.9 \%$. Only Black race was a statistically significant predictor in the model, $p<0.05$. Regression coefficients and standard errors can be found in Table 4 .

Table 4

Multiple regression results for patient satisfaction (PSQ-18 Total Score)

\begin{tabular}{|c|c|c|c|c|c|c|c|c|}
\hline \multirow[t]{2}{*}{ PSQ-18 } & \multirow[t]{2}{*}{$B$} & \multicolumn{2}{|c|}{$95 \%$ CI for $B$} & \multirow[t]{2}{*}{$S E B$} & \multirow[t]{2}{*}{$B$} & \multirow[t]{2}{*}{ Sig } & \multirow[t]{2}{*}{$R^{2}$} & \multirow[t]{2}{*}{$\overline{\operatorname{Adj} R^{2}}$} \\
\hline & & $L L$ & $U L$ & & & & & \\
\hline & & & & & & & .117 & .059 \\
\hline \multicolumn{9}{|l|}{ Model } \\
\hline Constant & 78.39 & 68.36 & 88.44 & 5.08 & & & & \\
\hline Age & -.024 & -.16 & .11 & .067 & -.032 & .720 & & \\
\hline \multicolumn{9}{|l|}{ Gender } \\
\hline Male & $0^{\mathrm{a}}$ & - & - & - & - & - & & \\
\hline Female & -2.62 & -7.07 & 1.84 & 2.25 & -.09 & .248 & & \\
\hline \multicolumn{9}{|l|}{ Education level } \\
\hline College & $0^{\mathrm{a}}$ & - & - & - & - & - & & \\
\hline Some College & -2.99 & -7.71 & 1.72 & 2.39 & -.12 & .212 & & \\
\hline High School & 1.15 & -4.12 & 6.41 & 2.67 & .05 & .668 & & \\
\hline$<$ High School & -1.48 & -7.80 & 4.84 & 3.12 & -.04 & .644 & & \\
\hline \multicolumn{9}{|l|}{ Race/ethnicity } \\
\hline White & $0^{\mathrm{a}}$ & & & & & & & \\
\hline Black & -5.75 & -10.22 & -1.28 & 2.26 & -.238 & $.012 *$ & & \\
\hline Hispanic & -.797 & -6.65 & 5.06 & 2.96 & -.025 & .788 & & \\
\hline Other & -5.76 & -12.29 & .753 & 3.30 & -.149 & .083 & & \\
\hline $\mathrm{MH}$ rating & -1.38 & -3.13 & .367 & .885 & -.150 & .121 & & \\
\hline Health rating & -.394 & -2.47 & 1.69 & 1.05 & -.036 & .708 & & \\
\hline
\end{tabular}

${ }^{a}$ Variable is reference in regression equation; $B$, unstandardized regression coefficient; $C I$, confidence interval; $L L$, lower limit; $U L$, upper limit; $S E B$, standard error of coefficient; $B$, standardized coefficient; $R^{2}$, coefficient of determination; Adj $R$, adjusted $R^{2}$; MH, mental health ${ }^{*} p<.05$ 


\section{Qualitative outcomes}

\section{Provider themes}

Twelve themes emerged from the provider qualitative data. See Table 5 for a detailed list of the themes, description and exemplar quotes. Several of the themes highlighted challenges within the integrated model: unclear roles/expectations of providers, providers not available or nearby for consultation/collaboration, communication issues, staff turnover/issues, time demands/pressure, working on improving processes, and feeling as if the business (e.g., financial) incentives outweighed patient centered care. For instance, a nurse provider remarked that it can be difficult for same day consultations to work: "At times we don't have the full staffing available. Like if we need to [get the] behavioral health person or dental person, they may be tied up with other patients."

At the same time, providers gave several recommendations for improvement, including more meetings among providers, in-house psychiatry, more staffing, referral specialists, optometry, X-ray, education, and more clinic hours. The positive themes included appreciating the one stop shop for patients, providing patient centered and holistic care, and overall reporting positive work experiences within the clinic. As one medical provider remarked, “...when it works, it works well.”

\section{Patient themes}

Nine themes emerged from the data. Table 6 features a detailed description along with exemplars. Feedback from the patients was overwhelmingly positive. They reported high satisfaction, positive interactions with the providers, affordability, health improvements, access to many resources, and acceptability of the one stop shop model. As one patient summed up their experience: "I'm very grateful to [the clinic]. I'm glad that it's in the community."

Problems with outside referrals were a consistent issue and many patients also reported infrastructure problems such as wait times and staff turnover. Suggested improvements included including other services such as additional therapy, additional hours of operation, and another location.

\section{Discussion}

The purpose of this study was to examine patient and provider perspectives of integrated care and reported levels of integration at an FQHC using a mixed methodology design. Overall, the providers and patients were generally satisfied with the model as evidenced by the surveys and qualitative results. However, there were some complexities to these data that were evident from the focus groups and interviews. Further, the extent of integration as reported by both patient and providers suggests potential areas for improvement, and by extension, highlights the challenges of implementing a complex system such as integration within community health clinics.

\section{Extent of integration}

Documenting the level and extent of integration occurring at a clinic is important in order to track model implementation and make improvements as necessary. In a fully integrated clinic, providers communicate and collaborate consistently, share the same space, facilities, and records, and work together as a team to provide care. ${ }^{20}$ Indeed, one of the hallmarks of integration is the warm hand-off: the same day introduction of another provider to provide clinical care or consultation. ${ }^{5,6}$ This is contrasted with co-located care, where providers may work at the same facility and may even share the same space, but may communicate only on certain shared patients and 


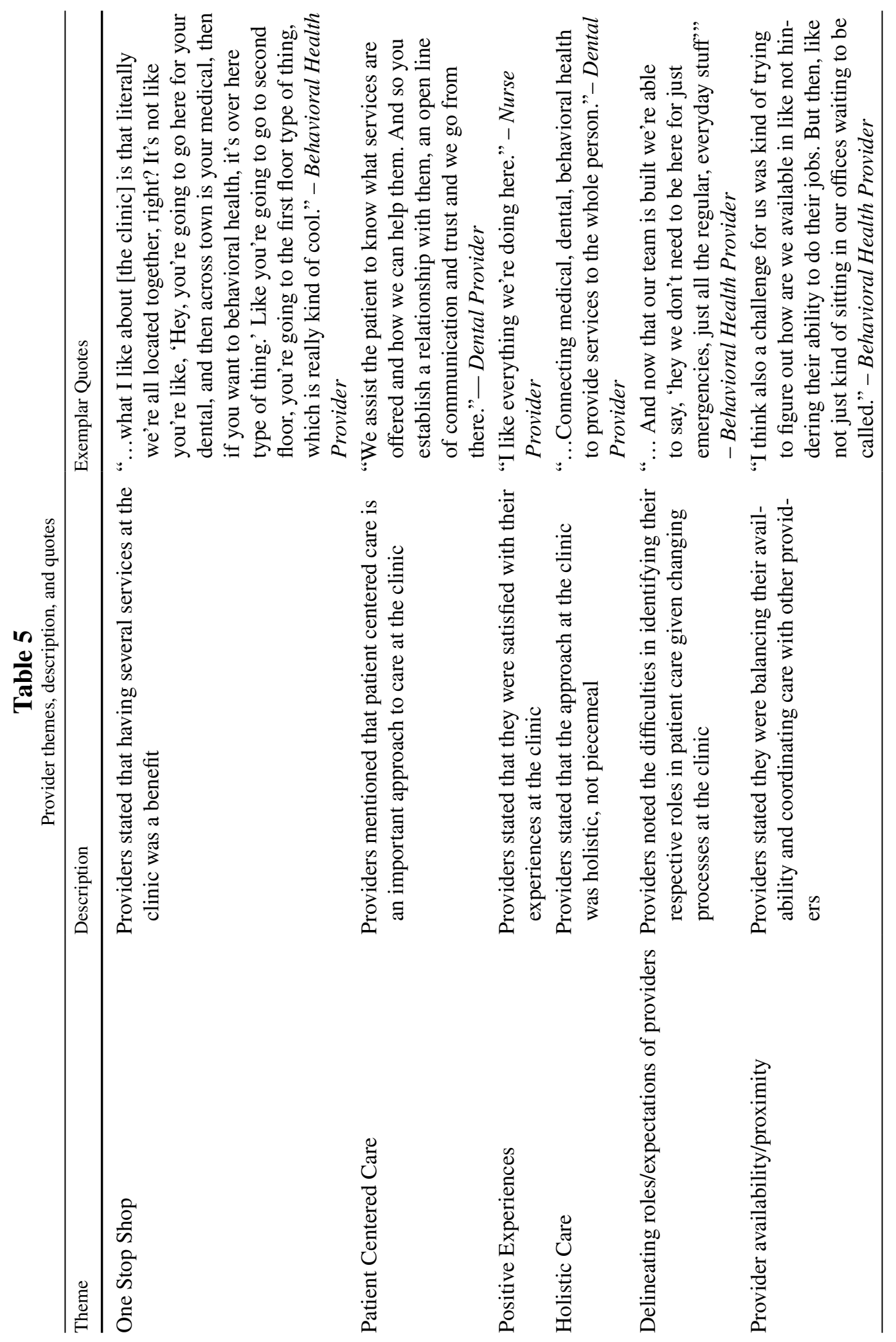




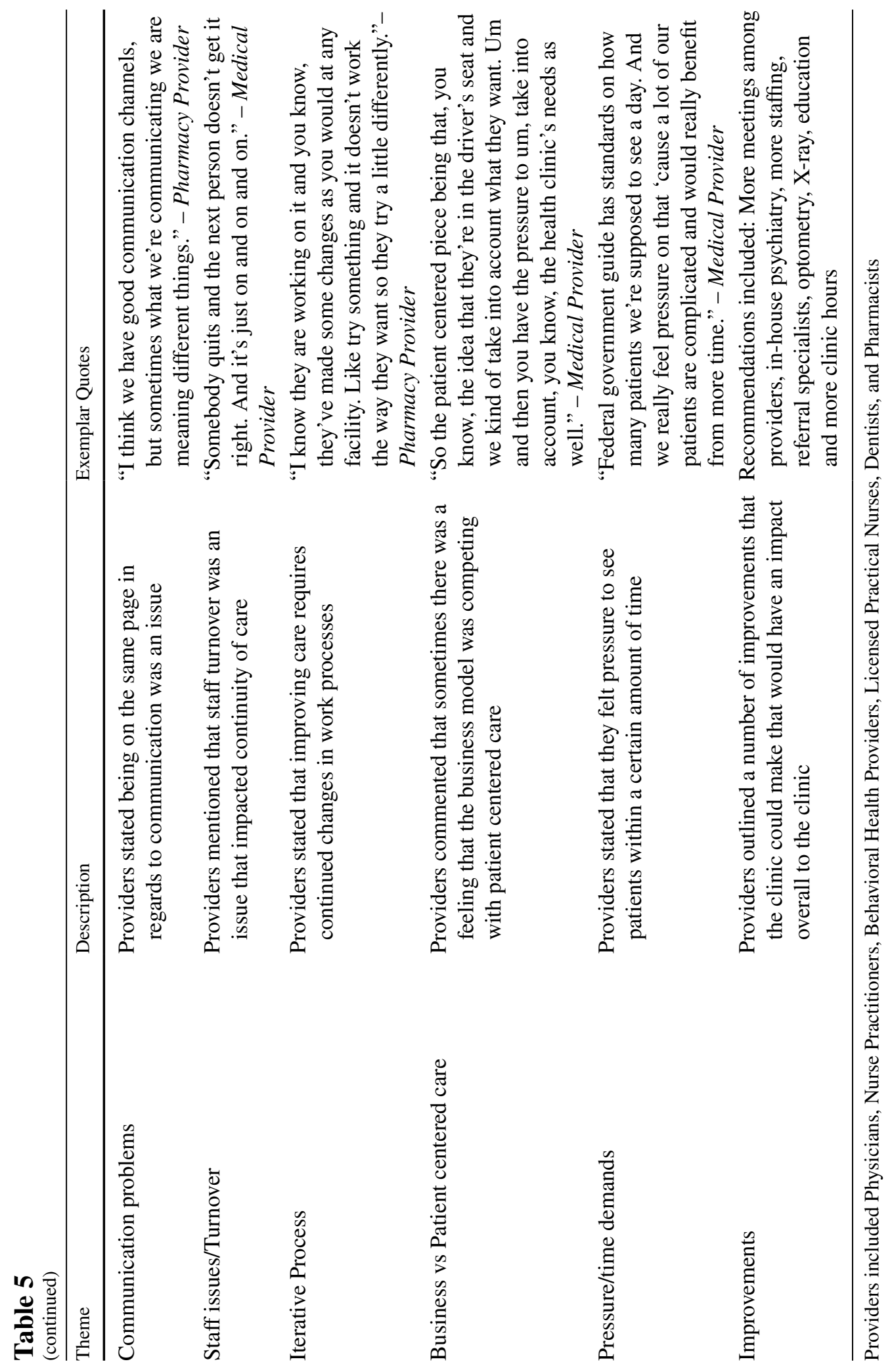




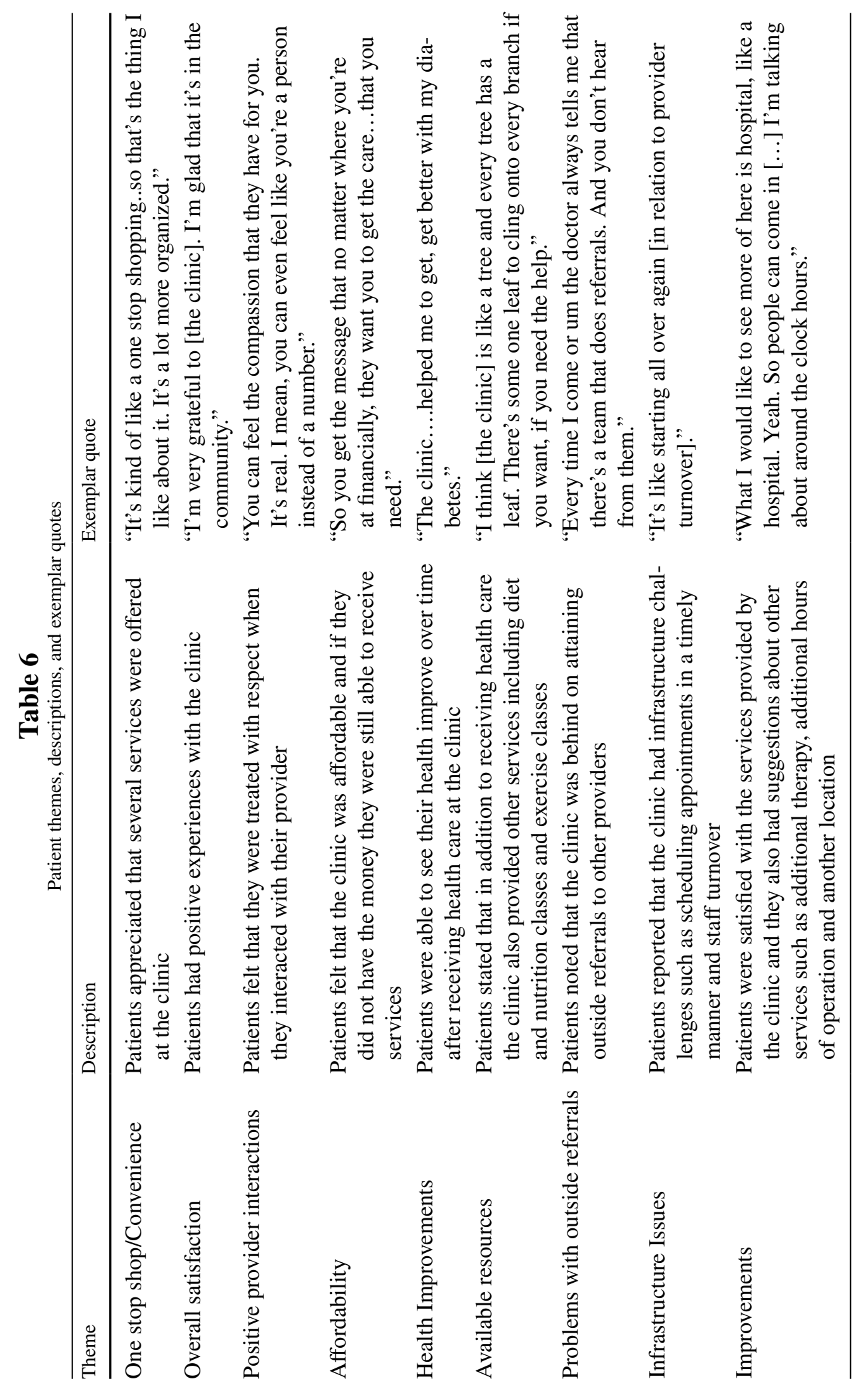


do not regularly involve each other on patient care. ${ }^{5,20}$ Clinics vary at what point they fall on the continuum of integration and it is often the case that newer clinics begin co-located, with the goal of moving towards full integration.

Results from the current evaluation suggest that the clinic is farther along the continuum of integration, with fairly frequent communication and collaboration and a little over a third of providers completing warm hand-offs "very often." This finding is noteworthy given that there was no formalized incentive to complete the warm hand-offs and providers had only been instructed to do so if there was an identified need. While medical and behavioral health are the most frequent collaborators (88\% each), dental was not too far behind (65\%), and pharmacy is called upon less frequently (around 35\%). The relatively lower involvement with pharmacy is not surprising given the typical role of the pharmacist (i.e., as a prescription service), and demonstrates a potential area for expansion -where the pharmacist is an active member of the health care team to enhance treatment recommendations and improve medication adherence. ${ }^{15}$ The patient report of integration mostly mirrored provider report, although patients reported accessing dental services slightly more frequently than behavioral health (28.5\% vs. $25.3 \%)$. This finding was unexpected given the higher rates of collaboration among medical and behavioral health providers; further it also suggests that the majority of patients are not accessing all of the services available. On the other hand, to what extent the patient population needs dentistry or behavioral health is unclear. A better measure of the reach of integration may involve screening for dentistry and behavioral health needs and tracking what percentage of positive screens access the needed service. Additionally, objective measures of integration taken from the electronic medical record (e.g., number of same day visits) would be an ideal method of documenting model implementation. Regardless, the self-report of both patients and providers suggest that while integration is occurring to an extent, there is still room for improvement.

Themes from the provider interviews may help to contextualize these findings: they reported that provider availability for collaboration or consultation was an issue, that the roles of different providers were unclear and that communication at times was difficult, that there were several time and financial pressures that made patient care difficult, and that staff turnover was frequent. Taken together, the qualitative data provide some insight as to what may be challenging about integration from the provider perspective. While some of the challenges discussed may not be inherent to integrated care itself (e.g., staff turnover at FQHCs is common), they more explicitly point to problems within the system that have the potential to be ameliorated. For instance, regarding the issue of provider availability, the clinic may look to ways to increase the physical proximity of collaborating providers or change scheduling to have certain providers on call for consultation at certain times.

These data should also be considered within the context of when and how integration occurred at the clinic. In January 2018, the clinic opened a 45,000 square foot facility that created space to bring in all integrated services (i.e., medical, dental, behavioral health, pharmacy, and expanded outreach) as well as the transition to a new integrated electronic health system. The clinic also hired new staff (about 30 full time employees) and trained them on the clinic's developing model of care. Data collection for this study spanned Spring through Fall 2019, approximately a year and a half after the clinic's rapid expansion. In other words, full scale integration is relatively new for the clinic and the data regarding the extent and level of integrated care may reflect both the growing pains and impressive ability to scale up integration quickly in a community health setting. However, it should be noted that the data were collected prior to the COVID-19 pandemic, which has undoubtedly impacted the ability to provide continuous and collaborative care for patients. ${ }^{21}$ While the data do not speak specifically to this context, one can surmise that the major changes that the clinic has faced during this time - pivoting to telehealth and mobile clinics, focusing on testing and vaccination clinics - would pose a significant challenge to integration. 


\section{Patient and provider satisfaction}

Patient and provider satisfaction were generally high, as assessed by both of the researcher-created scales and the PSQ-18. While satisfaction is a well-documented finding within integrated care what is unique about this study's finding is that this satisfaction was captured within an urban serving FQHC that provides comprehensive integrated services. ${ }^{7,8}$ The majority of the extant literature has focused on the integration of behavioral health and medical only, and often within academic medical centers or Veteran's Affairs centers. Further, many studies use author-created scales that are not empirically validated. The addition of the PSQ-18 as a psychometrically sound tool of patient satisfaction strengthens the overall findings.

A few unique satisfaction related findings are worth noting within both the survey and focus group data. First, while patients were generally happy with the care they received and voiced several benefits of integration and the care within the clinic, they reported several problems within the clinic including the outside referral process (i.e., accessing services outside of the medical home) and infrastructure challenges (e.g., staff turnover). Patients also reported several potential improvements to the clinic including more hours and more services provided.

Secondly, and most notably, Black patients reported poorer satisfaction than White, Hispanic, and other races/ethnicities on the PSQ-18. In some contexts, Black patients have reported lower satisfaction with health care, which may be related to experiences of racism and discrimination, mistrust of the health care system, and poor patient-provider relationships. ${ }^{22,23}$ This finding is particularly noteworthy within the current context of racial disparities exacerbated by the COVID-19 pandemic. COVID-19 cases and deaths have disproportionally affected communities of color. ${ }^{24}$ The interaction of systemic racism, social inequities, and a higher concentration of chronic health diseases (e.g., diabetes or hypertension) place Black communities at higher risk for poor outcomes. This notable difference in patient experiences within the clinic, albeit pre-pandemic, highlights the on-going need to address health care inequities at the systems level.

Provider data were also nuanced. While the researcher created satisfaction questionnaire suggested decent satisfaction, the qualitative data emphasized challenges more so than advantages. This discrepancy underscores the strength of a mixed methodology approach to evaluation. While providers appear to buy into the model and believe it is providing better care for patients, they are also reporting barriers and challenges to implementing the model - a more realistic view of integration at the ground level and more helpful feedback as it pertains to improving the system.

The patient and provider satisfaction data also highlight the need for on-going assessment of capturing experiences within integrated clinics. The current study was time-limited and focused - a cross-sectional view of the functioning of the clinic at that particular time. However, it would be useful for clinics to find alternative ways to capture anonymous feedback across time. Patient messaging within the electronic medical record, text messaging, flyers, or emails with anonymous links, are all potential methods that are both convenient and confidential. Electronic methods are easier to implement (and may be preferrable), but also require more resources to set up. Regardless of format, more frequent measures of patient feedback (potentially after each encounter if done electronically) from a larger pool of patients would provide a more accurate assessment of experiences within the clinic.

\section{Limitations}

There were a number of limitations in this study. First, the patient survey was a convenience sample that was conducted at the clinic. As a result of this convenience sample, it is unclear whether the results can be generalized to the population served by the clinic or to FQHCs broadly. Relatedly, although it was made clear that participation in the study was voluntary, the recruitment method of 
approaching patients while waiting for services could have inadvertently led some participants to feel pressure to complete the survey. Further, while results were confidential and anonymous, the patients may have given socially desirable answers that they were satisfied. Similarly, although the recruitment method of providers (i.e., recruiting during a lunch that was provided by the research team) is typical within healthcare settings, it is possible that providers may have felt reluctant to share their true perspectives on the clinic. Third, instrumentation of the survey may have been a potential problem. Several of the questions were designed by the research team specifically for the integrated care model given a lack of psychometrically valid satisfaction surveys. However, the Cronbach's alpha suggests that both patient and provider satisfaction surveys that were created by the research team had good-to-excellent internal consistency and were at least a reliable measure of satisfaction.

With regards to the qualitative data, it is unclear whether saturation for both provider and patients was reached, particularly with the patient population. Although meals and gift cards were provided, patient outreach was harder than anticipated. However, there was a noticeable repetition of themes while focus groups were occurring and so it is likely that focus groups may be reflective of the patient experience. Finally, it is important to note that the focus group targeted patients with diabetes only, and thus may not generalize to experiences of other patients within the clinic.

\section{Future directions}

This study provides preliminary evidence for satisfaction of an integrated FQHC that provides medical, behavioral health, dental, pharmacy, among other services. It also demonstrates the challenges inherent in implementing this model and the growing pains of attempting integration with several different service providers. These types of implementation studies are important first steps in integrated care research, but more is needed. For instance, future research should examine the differential impact of race/ethnicity on satisfaction in integrated care, as previous studies suggest this is an experience that many Black Americans face when seeking health care in general. This focus may be even more significant now given the impact of the pandemic on communities of color. Further, measuring the extent of integration and satisfaction with services are considered implementation outcomes which are important when researching a model of care, but are not the only pertinent variable of interest. ${ }^{7,25}$ How integration affects patient health outcomes is perhaps the most significant outcome and remains to be extensively empirically evaluated.

\section{Implications for Behavioral Health}

Given that the vast majority of individuals who need behavioral health services do not receive them, evaluating models of care that have the potential to improve access to, and quality of, behavioral health care is important. This study highlights the experiences of patients who receive integrated behavioral health services and of the clinicians who provide them, offering a snapshot of real-world practice. This type of evaluation can help to improve the delivery of integrated services, particularly those offered in FQHCs. Many health systems and clinics ultimately seek to achieve the Quadruple Aim of Health care: improve the quality of care delivered, decrease costs, improve patient outcomes, and increase the quality of a provider's work life. ${ }^{26}$ Addressing behavioral health in primary care - among other facets of health and social factors — is one promising way to contribute to the improved delivery of health care within the community. Indeed, given the devastating impact of the COVID-19 pandemic, integrated care is even more essential for improving access to high quality health care and reducing health disparities. 
Funding This project was supported by a grant from the Kansas Health Foundation.

\section{Declarations}

Conflict of Interest The authors declare that they have no conflict of interests.

\section{References}

1. Geiger HJ. The first community health centers: a model of enduring value. The Journal of Ambulatory Care Management. 2005;28(4):313-320.

2. Bureau of Primary Health Care. 2018 National Health Center Data. Health Resources and Services Administration. Available online at https://bphc.hrsa.gov/uds/datacenter.aspx. Accessed July 30, 2020.

3. Blount A. Integrated primary care: organizing the evidence. Families, Systems, \& Health. 2003; 21(2):121-133.

4. Croft B, Parish SL. Care integration in the patient protection and affordable care act: implications for behavioral health. Administration and Policy in Mental Health and Mental Health Services Research. 2013;40(4):258-263.

5. Robinson P J, Reiter JT. Behavioral Health Consultation and Primary Care: Guide to Integrating Services ( $2^{\text {nd }}$ ed.). New York: Springer, 2016.

6. Reiter JT, Dobmeyer AC, Hunter CL. The primary care behavioral health (PCBH) model: an overview and operational definition. Journal of Clinical Psychology in Medical Settings. 2018;25(2):109-126.

7. Hunter CL, Funderburk JS, Polaha J, et al. Primary care behavioral health (PCBH) model research: current state of the science and a call to action. Journal of Clinical Psychology in Medical Settings. 2018; 25(2):127-156.

8. Possemato K, Johnson EM, Beehler GP, et al. Patient outcomes associated with primary care behavioral health services: a systematic review. General Hospital Psychiatry. 2018;53:1-11.

9. Krupski A, West II, Scharf DM, et al. Integrating primary care into community health centers: impact on utilization and costs of health care. Psychiatric Services. 2016;67(11):1233-1239.

10. Bridges AJ, Villalobos BT, Anastasia EA, et al. Need, access, and reach of integrated care: a typology of patients. Family, Systems, and Health. 2017;35(2):193-206.

11. Druss BG, von Esenwein SA, Glick GE, et al. Randomized trial of an integrated behavioral health home: the health outcomes management and evaluation (HOME) study. American Journal of Psychiatry. 2017;174(3):246-255.

12. Rodriguez EM, Gulbas LE, George-Jones J, et al. Interdisciplinary perspectives on an integrated behavioral health model of psychiatry in pediatric primary care: a community-based participatory research study. Community Mental Health Journal. 2019; 55(4):569-577.

13. Bernstein J, Gebel C, Vargas C, et al. Integration of oral health into the well-child visit at federally qualified health centers: study of 6 clinics, August 2014-March 2015. Preventing Chronic Disease. 2016; 13:E58.

14. Jones E, Shi L, Hayashi AE, et al. Access to oral health care: the role of federally qualified health centers in addressing disparities and expanding access. American Journal of Public Health. 2014;103(3): 488-493.

15. Ray S, Lokken J, Whyte C, et al. The impact of a pharmacist-driven, collaborative practice on diabetes management in an Urban underserved population: a mixed method assessment. Journal of Interprofessional Care. 2020;34(1): 27-35.

16. Wallerstein N, Duran B, Minkler M, et al. Developing and maintaining partnerships with communities. In: BA Israel, E Eng, AJ Schulz, et al (eds). Methods in Community-Based Participatory Research for Health. San Francisco, CA, Jossey-Bass, A Wiley Imprint, 2005, pp. 31-51.

17. Marshall GN, Hays RD. The Patient Satisfaction Questionnaire Short Form (PSQ-18). Santa Monica, CA: RAND Corporation, 1994.

18. Thayaparan AJ, Mahdi E. The patient satisfaction questionnaire short form (PSQ-18) as an adaptable, reliable, and validated tool for use in various settings. Medical Education Online. 2013; 18: 21747.

19. Braun V, Clarke V. Using thematic analysis in psychology. Qualitative Research in Psychology. 2006; 3(2): 77-101.

20. Heath B, Wise Romero P, Reynolds KA. A Review and Proposed Standard Framework for Levels of Integrated Healthcare. Washington, DC: SAMHSA-HRSA Center for Integrated Health Solutions, 2013.

21. Cooper Z, De Saze Zerden L. How COVID-19 has impacted integrated care practice: lessons from the frontlines. Social Work in Health Care. 2021;60(2):146-156.

22. Barr DA. Race/ethnicity and patient satisfaction: using the appropriate method to test for perceived differences in care. Journal of General Internal Medicine. 2004;19(9): 937-943.

23. LaVeist TA, Nickerson KJ, Bowie JV. Attitudes about racism, medical mistrust, and satisfaction with care among African American and white cardiac patients. Medical Care Research and Review. 2000;57:146-161.

24. Gravlee CC. Systemic racism, chronic health inequities, and COVID-19: A syndemic in the making? American Journal of Human Biology. 2020;e23482.

25. Proctor E, Silmere H, Raghavan R, et al. Outcomes for implementation research: Conceptual distinctions, measurement challenges, and research agenda. Administration and Policy in Mental Health. 2011;38(2):65-76.

26. Bodenheimer T, Sinsky C. From triple to quadruple aim: Care of the patient requires care of the provider. Annals of Family Medicine. 2014;12(6):573-576.

Publisher's Note Springer Nature remains neutral with regard to jurisdictional claims in published maps and institutional affiliations. 